\title{
Electron Tomography of ZnO Nanoscale Tetrapods
}

\author{
S. Mehraeen, ${ }^{*}$ S. Kim, ${ }^{*}$ J. S. Lee, ${ }^{*}$ J. E. Evans, ${ }^{* *}$ and N. D. Browning*,*** \\ * Department of Chemical Engineering \& Materials Science, University of California, Davis, One \\ Shields Avenue, Davis, CA 95616 \\ ** Department of Molecular \& Cellular Biology, University of California, Davis, One Shields \\ Avenue, Davis, CA 95616 \\ *** Chemistry and Materials Science, Lawrence Livermore National Laboratory, Livermore CA \\ 94550, USA
}

Zinc oxide $(\mathrm{ZnO})$ is a unique material that exhibits properties such as semiconductivity, wear resistance, and microwave absorption. $\mathrm{ZnO}$ nanostructures are also very promising for applications in field emission displays and photonic devices operating in the blue and ultra violet spectral ranges, due to the wide band gap $(3.37 \mathrm{eV})$ and large exciton binding energy $(60 \mathrm{meV})$. The fabrication and related properties of $\mathrm{ZnO}$ nanostructures have therefore, attracted considerable attention. Among the multiple morphologies that have been produced are nanobelts, nanowires, tetrapod nanostructures, tabular nanostructures, nanobridges, and nanonails. Of these structures, it is the tetrapods that immediately appear to be of most interest. For example, $\mathrm{ZnO}$ tetrapod nanorods demonstrate field emission with turn-on field of $1.6 \mathrm{~V} / \mu \mathrm{m}$ and $\mathrm{ZnO}$ tetrapod nanorods/acrylic resin composite demonstrates both anti-electrostatic and anti-bactrial functions [1,2].

In the present research the three-dimensional (3D) structure of $\mathrm{ZnO}$ tetrapods ( $>99.99 \%$ purity) was investigated using Bright Field TEM, Bright Field STEM and Dark Field STEM tomography. The aim in using tomography to study the tetrapods is to evaluate the angles between the legs of the structure, thereby giving a measurement of the stress in the structure. High resolution imaging and electron energy loss spectroscopy (EELS) was also used to evaluate the structure and composition of the tetrapods. Combined with the 3-D reconstructions, the high resolution images and compositional analyses allow a complete picture of the nanostructures to be determined. A secondary aspect of this work is to evaluate the strengths and weaknesses of the various imaging methods to form the 3-D reconstructions.

The first set of images that were recorded in the analysis of the tetrapods were obtained with the Bright Field TEM approach. A series of 121 projections of the specimen were recorded over a tilt range of $-60^{\circ}$ to $+60^{\circ}$ on a JEOL 2100 field-emission TEM/STEM and were processed with the IMOD software package [3]. This software was used to track markers (here an individual tetrapod in the sample) through the 121 stacks to align them with respect to each other and yield a 3-D image. Figure 1A shows an image of an individual tetrapod in the specimen, which was later used for the series. The high resolution observation of the cross section of each leg in tetrapods confirms that they have wurtzite crystal structure and grow along the $<0001>$ direction in agreement with previous studies. The legs were typically $200-1000 \mathrm{~nm}$ long and $10-15 \mathrm{~nm}$ wide. The cross sectional view of a leg is shown in Figure 1B.

One of the problems associated with Bright Field TEM tomography for materials science is the presence of diffraction contrast. This is illustrated in figure 2, where a leg of the tetrapod appears to change contrast from low to high tilt. In terms of the reconstruction from the image the net effect is 
that there appears to be a composition variation that moves along the legs as a function of the tilt angle. Dark Field STEM of the tetrapod legs showed some bright zones in the legs, which appeared dark in Bright Field STEM imaging. This implies that there are some inhomogeneities in the composition of tetrapods like $\mathrm{Zn}$ as a precursor impurity and/or thickness variations. However, unlike the bright field case, these inhomegeneities did not move with tilt angle. This is a very important aspect of the dark field technique for this study since the quantity of impurities and structural defects in the tetrapods influence the properties of the material. The reconstruction of the tetrapods by dark field STEM will be discussed in detail in the presentation and the specific strengths and weaknesses of each method will be described.

\section{References}

[1] Y. H. Leung, A. B. Djurisic, J. Gao, M. H. Xie, W. K. Chan, Chem, Phys. Lett. 385 (2004) 155.

[2] Z. Zhou, W. Peng, S. Ke, H. Deng, J. Mat. Proc. Tech. 89 (1999) 415.

[3] J. R. Kremer, D. N. Mastronarde and J. R. McIntosh, J. Struc. Biol. 116 (1) (1996) 71.

[4] Y. Dai, Y. Zhang, Q. K. Li, C. W. Nan, Chem. Phys. Lett. 358 (2002) 83.
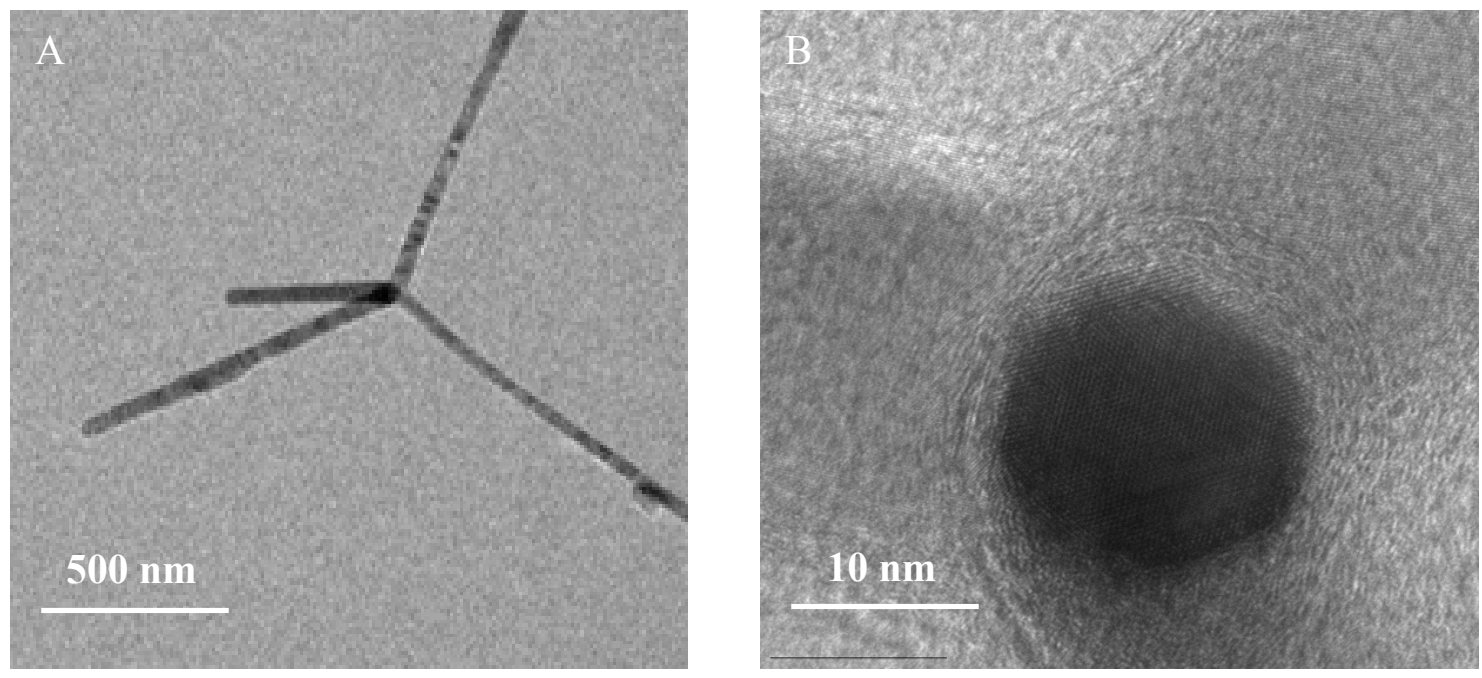

Fig. 1. A. TEM micrograph of an individual tetrapod. B. TEM micrograph of a tetropod's leg in its cross section view showing its hexagonal shape.
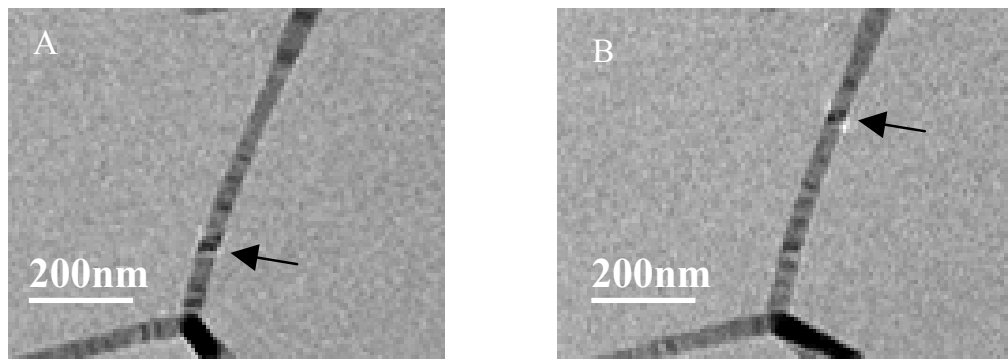

Fig 2. Movement of the dark zones in the legs of a tetrapod in different tilt angles.

A. Bright Field TEM in $5^{\circ}$. B. Bright Field TEM in $15^{\circ}$. 